\title{
Cryptanalysis of LowMC instances using single plaintext/ciphertext pair
}

\author{
Subhadeep Banik $^{1}$, Khashayar Barooti ${ }^{1}$, F. Betül Durak ${ }^{2}$, Serge Vaudenay ${ }^{1}$ \\ ${ }^{1}$ LASEC, École Polytechnique Fédérale de Lausanne, Lausanne, Switzerland \\ \{subhadeep.banik, khashayar . barooti, serge.vaudenay\}@epfl.ch \\ ${ }^{2}$ Robert Bosch LLC - Research and Technology Center - Pittsburgh PA, USA \\ durakfbetul@gmail.com
}

\begin{abstract}
Arguably one of the main applications of the LowMC family ciphers is in the post-quantum signature scheme PICNIC. Although LowMC family ciphers have been studied from a cryptanalytic point of view before, none of these studies were directly concerned with the actual use case of this cipher in PICNIC signature scheme. Due to the design paradigm of PICNIC, an adversary trying to perform a forgery attack on the signature scheme instantiated with LowMC would have access to only a single given plaintext/ciphertext pair, i.e. an adversary would only be able to perform attacks with data complexity 1 in a known-plaintext attack scenario. This restriction makes it impossible to employ classical cryptanalysis methodologies such as differential and linear cryptanalysis. In this paper we introduce two key-recovery attacks, both in known-plaintext model and of data complexity 1 for two variants of LowMC, both instances of the LowMC cryptanalysis challenge.
\end{abstract}

Keywords: LowMC · PICNIC Signature Scheme · MITM · 3-xor problem

\section{Introduction}

In recent years, a significant amount of attention has been drawn towards designing post-quantum cryptographic primitives, such as digital signature schemes. There have been several design ideas for quantum-secure signature schemes, some of which are based on lattice problems, supersingular isogenies or schemes providing information-theoretic security.

PICNIC $\left[\mathrm{CDG}^{+}{ }^{17}\right]$ is a highly tweakable signature scheme based on an MPC-in-head paradigm, currently in the third round of NIST post-quantum cryptography competition [nis]. The authors propose several different parameters for various security levels and applications. Instantiating PICNIC requires a hard to invert function which has low computational overhead when computed in a multi-party manner. This overhead relies heavily on the number of non-linear operations needed to compute the function, i.e. number of multiplications.

LowMC $\left[\mathrm{ARS}^{+} 15\right]$ is an efficient block-cipher tailored specifically for FHE and MPC usage, aiming to minimize the number of multiplications. LowMC uses a quadratic S-box operating on 3 bit inputs, and for each output bit of the S-box, a single multiplication is needed. The S-box is then followed by an affine layer, operating on the whole block, followed by a round-key addition.

The low multiplication count makes LowMC a fairly suitable choice for PICNIC instantiation. Both the LowMC instance and the MPC steps of PICNIC have multiple parameters to set for different use cases, but this also makes it challenging to select the optimal parameters, one of which is the number of rounds/S-boxes. As mentioned before, 
the computational overhead of performing a LowMC encryption in a multi-party manner is heavily dependent on the number of multiplications needed. Moreover, reducing the number of multiplications too much will lead to security issues, which makes it challenging to find a good parametrization to maximize efficiency without harming the security.

In ICISC 2015 Dobraunig et al. [DEM15] proposed an attack on LowMC family of block ciphers, based on cube attack strategies. The authors proposed an algorithm which successfully recovers the key of the round reduced version of the cipher, aiming for 80-bit security. Later in FSE 2018, Rechberger et al. [RST18] proposed a meet-in-the-middle style attack, based on possible output differentials, given an input differential, which affects the security of the variants of LowMCv2 with partial S-box layers drastically. In [LIM20] some results on LowMC were reported building on the techniques of [RST18], albeit with higher data complexities, which naturally do not apply to the PICNIC scenario. For a survey of key recovery attacks on LowMC, readers may check the survey done by Rechberger et al. [DKRS]. As mentioned, one of the main use cases of LowMC, is the PICNIC post quantum signature scheme. Due to PICNIC's algebraic composition, the scheme would be trivially forged by a key recovery attack on LowMC that uses only a single pair of plaintext/ciphertext. In other words only attacks with data complexity one directly affect the security of the signature scheme.

In May 2020, Rechberger et al. started a cryptanalysis challenge for LowMC keyrecovery, specifically for the PICNIC use case, meaning the attacks should be performed using a single ciphertext/plaintext pair in a known-plaintext model. In this paper we propose two attacks with data complexity one for two parameter sets of the LowMC challenge. Our attacks successfully break the two-round version of LowMC with full S-box layer, and the partial S-box variant with $0.8 \times\lfloor n / s\rfloor$ rounds, where $n$ denotes the block size, and $s$ denotes the number of S-boxes used in each round.

We continue by giving a brief high-level description of the PICNIC signature, and intuitively demonstrate why a data-complexity one key-recovery attack on LowMC cipher would lead to a PICNIC signature forgery.

\subsection{PICNIC Signature Scheme}

PICNIC signature is built using Fiat-Shamir transformation of a sigma protocol based on the MPC-in-head paradigm by Ishai et al. [IKOS07]. The high-level idea is as follows, imagine we have a multi-party computation of a function $f$. Each player has a share of the input $x$, and the output $y=f(x)$ is publicly known. The prover simulates all players and commits to all the states and transcripts. Later the verifier is allowed to corrupt a random subset of players, having access to their full state. Having this information in hand, the verifier can check whether the computation was done correctly from the corrupted players' perspective.

In the case of PICNIC this paradigm is instantiated using LowMC block cipher. Let $E(K, p t)$ be the LowMC encryption of the plaintext $p t$ using the key $K$. The function $f$ in the previous paradigm is instantiated as $E(*, p t)$ for a public plaintext $p t$. The plaintext/ciphertext pair $(p t, c t)$ is used as the public key of the signature scheme (verification key) and encryption key $K$ is used as the secret key (signing key). If an adversary can recover the encryption key given only a single ciphertext, plaintext pair $(c t, p t)$ i.e. the public key of the signature scheme, then in effect he computes the secret signing key. This allows him to forge a signature by following exactly the honest prover protocol with the recovered signing key. This demonstrates that a data complexity one key recovery attack on LowMC block cipher leads to a signature forgery on PICNIC.

The size of the signature and the efficiency of the PICNIC signing algorithm heavily rely on the number of the multiplications (and gates) used in the encryption circuit. This has driven some interest towards finding an optimal number of and gates, to keep the desired security level and to provide the best level of efficiency, and recently Rechberger et 


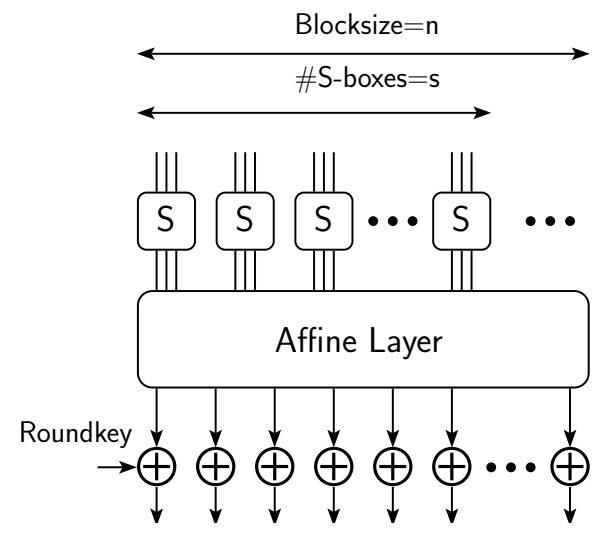

Figure 1: LowMC Round Function

al. announced a cryptanalysis challenge for LowMC family of block ciphers, specifically for the PICNIC use case, i.e. only one plaintext ciphertext provided to the attacker.

\subsection{Contribution and Organization of the Paper}

In this paper we introduce two cryptanalysis results for two instances of the LowMC cryptanalysis challenge, namely the 2 round LowMC with full S-box layer and the partial S-box layer with $0.8 \times\lfloor n / s\rfloor$ number of rounds. Both attacks are performed in a known plaintext/ciphertext attack model (KPA/KCA), and both attacks have data complexity 1. These restrictions make the task fairly hard, as the classical cryptanalysis methods such as linear and differential cryptanalysis can not be employed. In this paper, we first show how to efficiently linearize the LowMC S-box by guessing only one quadratic expression in the S-box inputs. Leveraging this fact we describe a series of attacks on some instances of LowMC given in the cryptanalysis challenge. Our results are summarized in Table 1.

In section 2, we describe the algebraic form of the LowMC round function and furnish some details of the LowMC cryptanalysis challenge. Section 3 demonstrates an efficient method to linearize the LowMC S-box which will be used later. Section 4 introduces a key-recovery attack on 2-round full S-box layer and partial S-box with $0.8 \times\lfloor n / s\rfloor$ round variants of LowMC. In Section 5, we introduce a meet in the middle attack on the same variants of LowMC, and in Section 6 we demonstrate how this approach can be optimized using the 3 -xor problem by a factor of $\sqrt{n / 2}$. We conclude the paper in Section 7 .

\section{The LowMC challenge}

The LowMC round function is a typical SPN construction given in Figure 1. It consists of an $n$-bit block undergoing a partial substitution layer consisting of $s$ S-boxes where $3 s \leq n$. It is followed by an affine layer which consists of multiplication of the block with an invertible $n \times n$ matrix over $\mathbb{F}_{2}$ and addition with an $n$-bit round constant. Finally the block is xored with the roundkey which is again the product of the $n$-bit master secret key $K$ with an $n \times n$ invertible matrix. As in most SPN constructions, a plaintext is first xored with a whitening key which for LowMC is simply the secret key $K$, and the round functions are executed $r$ times to give the ciphertext. From the point of view of cryptanalysis, we note that the design is completely known to the attacker, i.e. all the matrices and constants used in the round function and key update are known.

The Low MC challenge specifies 9 challenge scenarios for key recovery given only 1 plaintext-ciphertext pair, i.e. the data complexity $d=1$. 
- $n=128, \quad s=1$

- $n=128, \quad s=10$

- $n=129, \quad s=43$ (full S-box layer)

- $n=192, \quad s=1$

- $n=192, \quad s=10$

- $n=192, \quad s=64$ (full S-box layer)

- $n=256, \quad s=1$

- $n=256, \quad s=10$

- $n=255, \quad s=85$ (full S-box layer)

The number of rounds $r$ for instances with the full S-box layer is either 2, 3, or 4 and for instances with a partial S-box layer can vary between $0.8 \times\left\lfloor\frac{n}{s}\right\rfloor,\left\lfloor\frac{n}{s}\right\rfloor$ and $1.2 \times\left\lfloor\frac{n}{s}\right\rfloor$. The key length $k$ for all instances is $n$ bits. Note that in general instantiations of LowMC, the key size and block size are not the same. The whitening key and all the round keys are extracted by multiplying the master key with full rank matrices over $G F(2)$. However for all the instances of LowMC used in the LowMC challenge the block size and key size are the same. This being so, the lengths of the master key, whitening key and all the subsequent round keys are the same. Effectively, this makes all these keys related to each other by multiplication with an invertible matrix over $G F(2)$. Thus all round keys can be extracted by multiplying the whitening key with an invertible matrix. So for all practical purposes used in this paper, the whitening key can also be seen as the master secret key. This is true since given any candidate whitening key, all round keys can be generated from it, and thus given any known plaintext-ciphertext pair, it is possible to verify if that particular candidate key has been used to generate the corresponding PT/CT pair. As such we use the terms master key/whitening key interchangeably.

\section{Preliminaries}

The algebraic forms of the 3 output bits of the S-box $S$ used in LowMC are given by the following expressions:

$$
\begin{aligned}
& s_{0}=x_{0}+x_{1} \cdot x_{2}, \\
& s_{1}=x_{0}+x_{1}+x_{0} \cdot x_{2}, \\
& s_{2}=x_{0}+x_{1}+x_{2}+x_{0} \cdot x_{1}
\end{aligned}
$$

Similarly the inverse S-box $S^{-1}$ is given by the following expressions

$$
\begin{aligned}
& t_{0}=x_{0}+x_{1}+x_{1} \cdot x_{2}, \\
& t_{1}=x_{1}+x_{0} \cdot x_{2}, \\
& t_{2}=x_{0}+x_{1}+x_{2}+x_{0} \cdot x_{1}
\end{aligned}
$$

Let us for example take $f$ to be the majority function computed on the inputs of the 3 input bits, i.e. $f=x_{0} \cdot x_{1}+x_{1} \cdot x_{2}+x_{0} \cdot x_{2}$. Then the expressions of the S-box can be rewritten as

$$
\begin{aligned}
& s_{0}=f \cdot\left(x_{1}+x_{2}+1\right)+x_{0}, \\
& s_{1}=f \cdot\left(x_{0}+x_{2}+1\right)+x_{0}+x_{1}, \\
& s_{2}=f \cdot\left(x_{0}+x_{1}+1\right)+x_{0}+x_{1}+x_{2}
\end{aligned}
$$


Table 1: Summary of results. Note for the Linearization+MITM (+3-xor) approaches the complexity is given in "evaluations of a quadratic expression". For the Linearization only approach, the complexity is in "number of Gaussian eliminations." * As explained in Section 4, these are best case complexities that occur for around $29 \%$ of the LowMC instances.

\begin{tabular}{|c|c|c|c|c|c|c|}
\hline Instance & $n$ & $s$ & $r$ & Type of Attack & Complexity & Section \\
\hline \multirow[t]{3}{*}{ Full S-box layer } & 129 & 43 & \multirow[t]{3}{*}{2} & \multirow[t]{3}{*}{ Linearization } & $2^{86}$ & \multirow[t]{3}{*}{4} \\
\hline & 192 & 64 & & & $2^{128}$ & \\
\hline & 255 & 85 & & & $2^{170}$ & \\
\hline \multirow[t]{3}{*}{ Partial Sbox layer } & 128 & 1 & \multirow[t]{3}{*}{$0.8 \times\left\lfloor\frac{n}{s}\right\rfloor$} & \multirow[t]{3}{*}{ Linearization } & $2^{102}$ & \multirow[t]{3}{*}{4} \\
\hline & 192 & 1 & & & $2^{154}$ & \\
\hline & 256 & 1 & & & $2^{205}$ & \\
\hline \multirow[t]{3}{*}{ Partial Sbox layer } & 128 & 10 & \multirow[t]{3}{*}{$0.8 \times\left\lfloor\frac{n}{s}\right\rfloor$} & \multirow[t]{3}{*}{ Linearization } & $2^{100}$ & \multirow[t]{3}{*}{4} \\
\hline & 192 & 10 & & & $2^{150}$ & \\
\hline & 256 & 10 & & & $2^{200}$ & \\
\hline \multirow[t]{3}{*}{ Full S-box layer } & 129 & 43 & \multirow[t]{3}{*}{2} & \multirow[t]{3}{*}{ Linearization + MITM } & $2^{109}$ & \multirow[t]{3}{*}{5} \\
\hline & 192 & 64 & & & $2^{161}$ & \\
\hline & 255 & 85 & & & $2^{214}$ & \\
\hline \multirow[t]{3}{*}{ Partial Sbox layer } & 128 & 1 & \multirow[t]{3}{*}{$0.8 \times\left\lfloor\frac{n}{s}\right\rfloor$} & \multirow[t]{3}{*}{ Linearization+ MITM } & $2^{124}$ & \multirow[t]{3}{*}{$5^{*}$} \\
\hline & 192 & 1 & & & $2^{186}$ & \\
\hline & 256 & 1 & & & $2^{248}$ & \\
\hline \multirow[t]{3}{*}{ Partial Sbox layer } & 128 & 10 & \multirow[t]{3}{*}{$0.8 \times\left\lfloor\frac{n}{s}\right\rfloor$} & \multirow[t]{3}{*}{ Linearization + MITM } & $2^{124}$ & \multirow[t]{3}{*}{$5^{*}$} \\
\hline & 192 & 10 & & & $2^{186}$ & \\
\hline & 256 & 10 & & & $2^{248}$ & \\
\hline \multirow[t]{3}{*}{ Full S-box layer } & 129 & 43 & \multirow[t]{3}{*}{2} & \multirow[t]{3}{*}{ Linearization + MITM+3-xor } & $2^{106}$ & \multirow[t]{3}{*}{6} \\
\hline & 192 & 64 & & & $2^{158}$ & \\
\hline & 255 & 85 & & & $2^{211}$ & \\
\hline
\end{tabular}

This means that if we guess the value of the single expression $f(0$ or 1$)$, then the entire S-box becomes an affine function in the input bits. The same holds for the inverse S-box. In fact we can replace $f$ with any balanced 3-variable Boolean function of degree 2, and still get the same results as we prove in the following lemma.

Lemma 1. Consider the LowMC S-box $S$ defined over the input bits $x_{0}, x_{1}, x_{2}$. If we guess the value of any 3-variable quadratic Boolean function $f$ which is balanced over the input bits of the S-box, then it is possible to re-write the S-box as affine function of its input bits.

Proof. The general expression for a 3 variable quadratic Boolean function is

$$
f=A+B x_{0}+C x_{1}+D x_{2}+E x_{0} \cdot x_{1}+F x_{1} \cdot x_{2}+G x_{0} \cdot x_{2} .
$$

The only non-linear terms in the expression of the LowMC S-box are $x_{0} \cdot x_{1}, x_{1} \cdot x_{2}, x_{0} \cdot x_{2}$. Thus if there exists a Boolean function of the above form, which when multiplied with different linear functions can produce each of the terms $x_{0} \cdot x_{1}, x_{1} \cdot x_{2}, x_{0} \cdot x_{2}$, then we are done. Thus the necessary and sufficient conditions required to achieve the above is to prove the existence of 3 affine Boolean functions $g_{i}=a_{i} x_{0}+b_{i} x_{1}+c_{i} x_{2}+d_{i}, \forall i \in[0,2]$, such that

$$
\begin{aligned}
& f \cdot g_{0}=x_{0} \cdot x_{1}+l_{0}\left(x_{0}, x_{1}, x_{2}\right) \\
& f \cdot g_{1}=x_{1} \cdot x_{2}+l_{1}\left(x_{0}, x_{1}, x_{2}\right) \\
& f \cdot g_{2}=x_{0} \cdot x_{2}+l_{2}\left(x_{0}, x_{1}, x_{2}\right)
\end{aligned}
$$

where $l_{0}, l_{1}, l_{2}$ are some affine functions on $x_{0}, x_{1}, x_{2}$. If these functions $g_{i}$ exist, we can write each of the three output bits of the LowMC S-box as

$$
x_{0}+f \cdot g_{1}+l_{1}, x_{0}+x_{1}+f \cdot g_{2}+l_{2}, x_{0}+x_{1}+x_{2}+f \cdot g_{0}+l_{0}
$$


So in order for the first equation to be satisfied, we need that the product of $f$ and $g_{0}$ produces coefficients $0,1,0,0$ for the terms $x_{0} \cdot x_{1} \cdot x_{2}, x_{0} \cdot x_{1}, x_{1} \cdot x_{2}, x_{0} \cdot x_{2}$ respectively. In matrix form this can be written as $\mathbf{M} \cdot\left[a_{0}, b_{0}, c_{0}, d_{0}\right]^{T}=[0,1,0,0]^{T}$, where

$$
\mathbf{M}=\left[\begin{array}{cccc}
F & G & E & 0 \\
C+E & B+E & 0 & E \\
0 & D+F & C+F & F \\
D+G & 0 & B+G & G
\end{array}\right]
$$

Similarly the other 2 equations can be written as $\mathbf{M} \cdot\left[\begin{array}{ll}a_{1}, & b_{1}, c_{1}, d_{1}\end{array}\right]^{T}=[0,0,1,0]^{T}$ and $\mathbf{M} \cdot\left[a_{2}, b_{2}, c_{2}, d_{2}\right]^{T}=[0,0,0,1]^{T}$. It is therefore clear that for the equations to have a solution we need $\mathbf{M}$ to be invertible. Since the number of 3 -variable quadratic Boolean functions $f$ is just $2^{7}$, we can perform the following small computer exercise: we can construct the matrix $\mathbf{M}$ for each function $f$ and test whether it is invertible or not. We found that all functions $f$ for which $\mathbf{M}$ is invertible, are exactly the functions that are balanced.

For example, if we take $f=s_{0}=x_{0}+x_{1} \cdot x_{2}$, the S-box functions can be written as

$$
\begin{aligned}
& s_{0}=f, \\
& s_{1}=f \cdot\left(x_{2}+1\right)+x_{1}, \\
& s_{2}=f \cdot\left(x_{1}+1\right)+x_{1}+x_{2}
\end{aligned}
$$

\section{Cryptanalysis by Linearization}

The first technique to break LowMC by linearization is for instances for which the total number of S-boxes is less than the key length. This occurs for the following cases

1. All instances of full S-box layer with number of rounds $=2$.

2. All instances of partial S-box layer with number of rounds $=0.8 \times\left\lfloor\frac{n}{s}\right\rfloor$.

The idea is as follows. We guess the value of the majority function at the input of all the S-boxes in the encryption circuit. When we do so the expression relating the plaintext and ciphertext becomes a linear expression in the key variables, i.e. of the form

$$
A \cdot\left[k_{0}, k_{1}, \ldots, k_{n-1}\right]^{T}=\text { const }
$$

where $A$ is an $n \times n$ matrix over $G F(2)$. Thus the key can be found using Gaussian elimination. After this a wrong key can be discarded by simply recalculating the encryption function with the derived key and plaintext and checking if the result equals the given ciphertext or not. Of course, we need not compute the full encryption: a key can be discarded as soon as the majority function computed at the input of one of the s-boxes differs from the value used to linearize the circuit. If the total number of s-boxes in the circuit is $t$, then the worst case complexity of the process is $2^{t}$ gaussian eliminations calculations. For example this is $2^{86}$ for the LowMC instance with $n=129, s=43, r=2$. However note that there is an added cost in this process. For any guess of the majority values, the matrix $A$ computed above may not necessarily be invertible. If the dimension of the kernel of the matrix $A$ is $d_{A}$, then we can see that $O\left(2^{d_{A}}\right)$ keys would satisfy any equation of the form $A \cdot K=$ const. Thus the verification would require running the verification for $2^{d_{A}}$ candidate keys. Moreover, we did not find any easy way to find a closed form for any bound on $d_{A}$. 


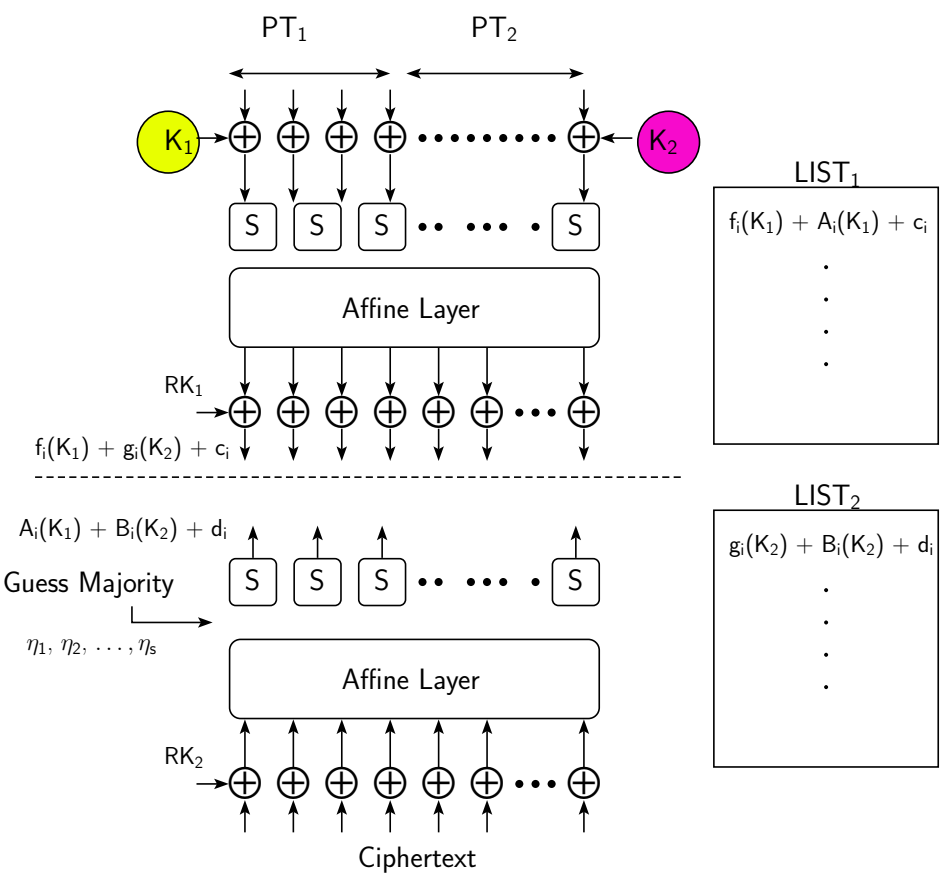

Figure 2: Meet in the Middle

\section{Meet in the Middle approach}

\subsection{2 round full S-box layer}

The complexity of the attack in the previous section was measured in terms of number of Gaussian eliminations. Even while bypassing the Gaussian Elimination method, the algorithm will still require an additional computational step (evaluating all elements in the kernel of $A$ ) and there is no easy way of finding a closed form of its value/lower bound. In this section we present attacks whose complexity is measured in much simpler and more tangible metric: "number of evaluations of a quadratic expression in keybits". We describe a meet in the middle approach for the two-round variant of LowMC. The idea is to first split the key into two parts $K_{1}=\left[k_{0}, \ldots, k_{t-1}\right]^{T}$ and $K_{2}=\left[k_{t}, \ldots, k_{n-1}\right]^{T}$, each of around $t \approx \frac{n}{2}$ bits. By guessing the majority bits (or any other balanced quadratic function) of the second layer S-box we can make the second round linear as described above. After this it is possible to adopt a meet in the middle approach, by guessing first the $K_{1}$ value and making a list based on each guess. We later independently guess $K_{2}$ and creating a list based on the guessed values and search for a collision in the obtained lists.

The idea is as follows. As proven in Lemma 1, if we know the value of a balanced quadratic boolean function in the input bits of each Sbox, i.e. the majority, we can write the S-box as an affine function in the input bits. The same argument holds for the inverse S-box (since the inverse S-box is also a quadratic permutation over $\{0,1\}^{3}$ ). Again let us denote by $R_{1}, R_{2}$ the first and second round functions i.e. $R_{1}\left(p t+R K_{0}, R K_{1}\right)=x$ and $R_{2}\left(x, R K_{2}\right)=c t$, where $x$ denotes the $n$-bit input to the second round and $R K_{1}, R K_{2}$ denotes the first, second round keys, respectively, which are of course linear functions of the original key $K=R K_{0}$. As shown in Figure 2, we start with the ciphertext backwards and try to reach the state at the input to the second round. To do this we first perform the inverse affine function operation on the vector $c t \oplus R K_{2}$ (where $R K_{2}$ is expressed in terms of $K_{1}$ and $K_{2}$ ). Thereafter we guess the $s$ majority bits $\eta_{1}, \ldots, \eta_{s}$ at the input of the second round inverse S-boxes to linearize $R_{2}$. After this, each bit of $x$ can be written 
as an affine function of the key and the ciphertext. In fact denoting each bit of $x$ as $x_{i}$, we can further write $x_{i}=A_{i}\left(K_{1}\right)+B_{i}\left(K_{2}\right)+d_{i}, \forall i \in[0, n-1]$, where each $A_{i}, B_{i}$ are linear functions over $K_{1}, K_{2}$ and $d_{i}$ is a single bit constant.

Similarly it is possible to compute $x$ from the plaintext in the forward direction. Even if we do not guess the majority of the first round s-boxes, $K_{1}$ and $K_{2}$ can be chosen such that the bits of $K_{1}$ and $K_{2}$ are never multiplied in the first round function. For example for $n=129, K_{1}$ can be taken to be the first $t=3 \times\lfloor s / 2\rfloor=63$ bits of the key and $K_{2}$ to be the remaining 66 bits. The only source of non-linearity in the first round are the S-boxes, and each S-box either gets the bits of $K_{1}$ or $K_{2}$ as inputs and so $K_{1}$ and $K_{2}$ are not mixed in a multiplicative sense in this round. This being the case, after the affine layer and addition of $R K_{1}$, each bit $x_{i}$ can be written as $f_{i}\left(K_{1}\right)+g_{i}\left(K_{2}\right)+c_{i}$ where each $f_{i}, g_{i}$ are at most quadratic functions over $K_{1}, K_{2}$ and $c_{i}$ is a single bit constant. Given the equality $x_{i}=f_{i}\left(K_{1}\right)+g_{i}\left(K_{2}\right)+c_{i}=A_{i}\left(K_{1}\right)+B_{i}\left(K_{2}\right)+d_{i}$, we can rearrange the terms to get

$$
f_{i}\left(K_{1}\right)+A_{i}\left(K_{1}\right)+c_{i}=g_{i}\left(K_{2}\right)+B_{i}\left(K_{2}\right)+d_{i}, \forall i \in[0, n-1]
$$

We are now ready to state the attack. Let the plaintext be $p t=\left[p t_{0}, p t_{1} \ldots, p t_{n-1}\right]$, and $c t=\left[c_{0}, c_{1}, \ldots, c_{n-1}\right]$ be the corresponding ciphertext. Take $t=3 \times\lfloor s / 2\rfloor \approx \frac{n}{2}$. We proceed as follows:

1. Calculate the functional forms of $f_{i}, g_{i}$ and $c_{i}$ for all $i \in[0, n-1]$.

2. Guess the values $\eta_{1}, \ldots, \eta_{s}$. This step is done $2^{s}$ times in the worst case.

- Compute $A_{i}, B_{i}, d_{i}$ for all $i \in[0, n-1]$ using the guessed values.

- For all possible values of $K_{1}$, create a hash table LIST $_{1}$ indexed by the $n$-bit vector $\left[f_{i}\left(K_{1}\right) \oplus A_{i}\left(K_{1}\right) \oplus c_{i}\right], \forall i \in[0, n-1]$. We need $2^{t}$ operations in this step.

- For all possible values of $K_{2}$, create a hash table LIST $_{2}$ indexed by the $n$-bit vector $\left[g_{i}\left(K_{2}\right) \oplus B_{i}\left(K_{2}\right) \oplus d_{i}\right], \forall i \in[0, n-1]$. We need $2^{n-t}$ operations in this step.

- Find a collision between $\operatorname{LIST}_{1}$ and $\mathrm{LIST}_{2}$.

- When a collision is found for $K_{1}$ and $K_{2}$ check if the majority bits are consistent with the guess of the key. If yes, this key is in fact the encryption key. Otherwise try another guess of $\eta_{1}, \ldots, \eta_{s}$.

In practice, 2 hash tables are not necessary. The attacker can insert each new vector of LIST $_{1}$ and LIST $_{2}$ into a single hash table and wait until a collision between elements of LIST $_{1}$ and LIST 2 is found. For each set of majority guesses, the complexity of the attack is dominated by finding a collision between two lists of length $2^{t}$ and $2^{n-t}$ each. So for $n=129$, we can take $t=63$ (key bits added before the first $21 \mathrm{~S}$-boxes) and $n-t=66$. The total complexity of the attack is $O\left(2^{s} \times\left(2^{t}+2^{n-t}\right)\right)$, which for the $n=129$ bit version is around $2^{43+66}=2^{109}$.

\subsection{MITM on partial S-box layers}

In order to perform a MITM on the partial S-box layer instances of LowMC, we rearrange the first $r_{1}$ and final $r_{3}$ rounds so that the total number of different key bits involved in these rounds is $3 s$ per round. The transformations are shown in Figures 3, 4 and are similar to the ones used in [RST18]. In fact the transform used in the backward direction (see Fig 4) is exactly same as the one used in [RST18, Fig.1]. The idea is that the affine layer and key addition are interchangeable. Since if $L$ is a linear function, we 


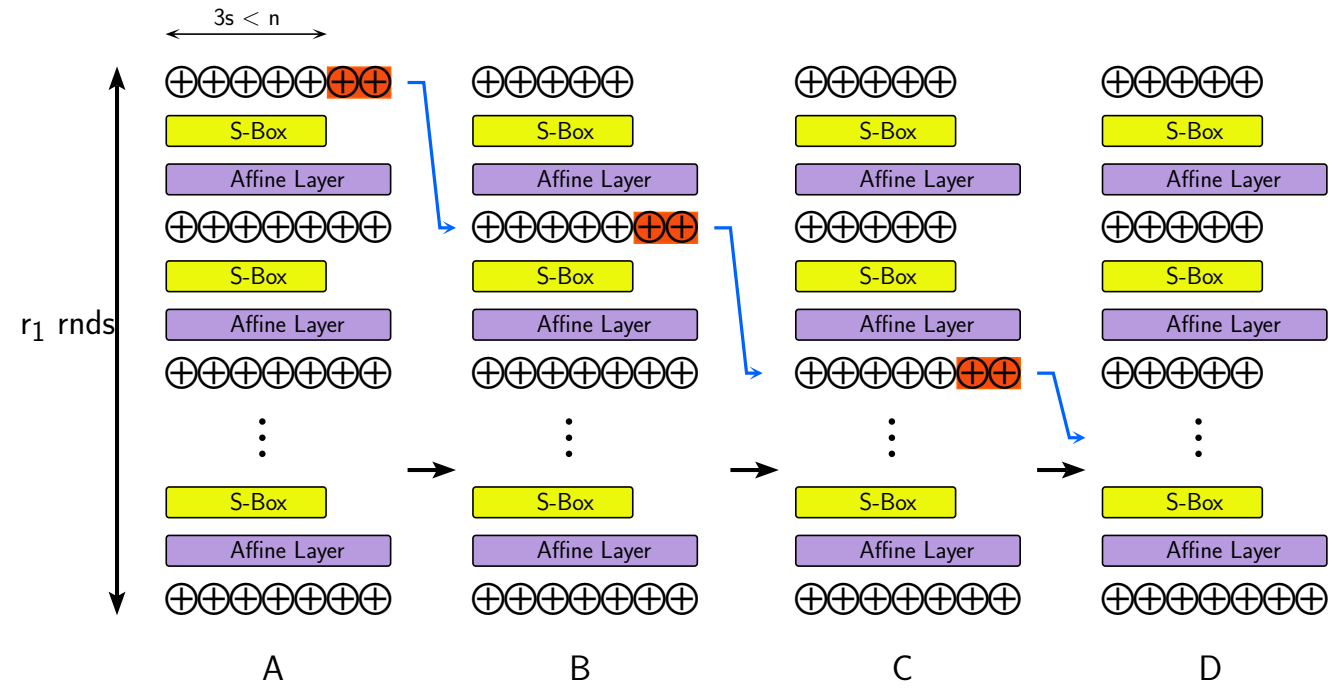

Figure 3: Transforming the round function in the first $r_{1}$ rounds. From $\mathrm{A} \rightarrow \mathrm{B}$, the key material not added to bits input to the S-box in round 1 (shown in orange background) are carried to the next round, through the affine layer and merged with the round key in round $2 . \mathrm{B} \rightarrow \mathrm{C} \rightarrow \mathrm{D}$ do the same from the second round onwards.

have $L(x)+K=L\left(x+L^{-1}(K)\right)$ and similarly $L(x+K)=L(x)+L(K)$. Hence the key addition can be moved before or after the affine layer as required, by multiplying the round key by the appropriate matrix. Fig 3 further shows how to transform the first $r_{1}$ rounds.

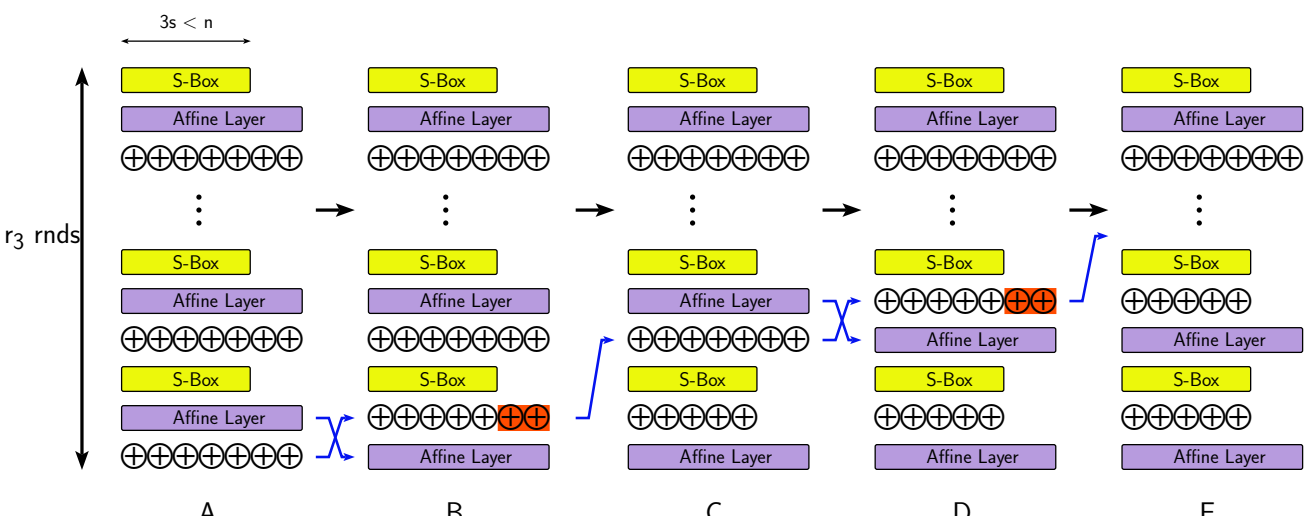

Figure 4: Transforming the round function in the final $r_{3}$ rounds. $\mathrm{A} \rightarrow \mathrm{B}$ flips the order of the last round Affine layer and round key xor. $\mathrm{B} \rightarrow \mathrm{C}$ takes the bits of the last round key that are not added to S-box outputs (shown in orange background), and brings them back by 1 round and merges it with the penultimate round key. $\mathrm{C} \rightarrow \mathrm{D}$ flips the order of the Affine layer and round key of the penultimate round, and D $\rightarrow$ E generalizes the process from this point onwards.

We partition the $r=r_{1}+r_{2}+r_{3}$ rounds of LowMC into the first $r_{1}$, middle $r_{2}$ and final $r_{3}$ rounds, and further transform the first $r_{1}$ and the final $r_{3}$ rounds so that each round has only $3 s$ keybits. If $r_{1}=r_{3}=\left\lfloor\frac{n}{6 s}\right\rfloor$, then there are a total of $n$ keybits in these rounds. Naming these keybits as $\kappa_{0}, \kappa_{1}, \ldots, \kappa_{n-1}$. Let us assume that these $n$ keybits result from linearly independent expressions on the master key bits (in the next subsection we will see what happens when this is not the case). Then it is not difficult to 


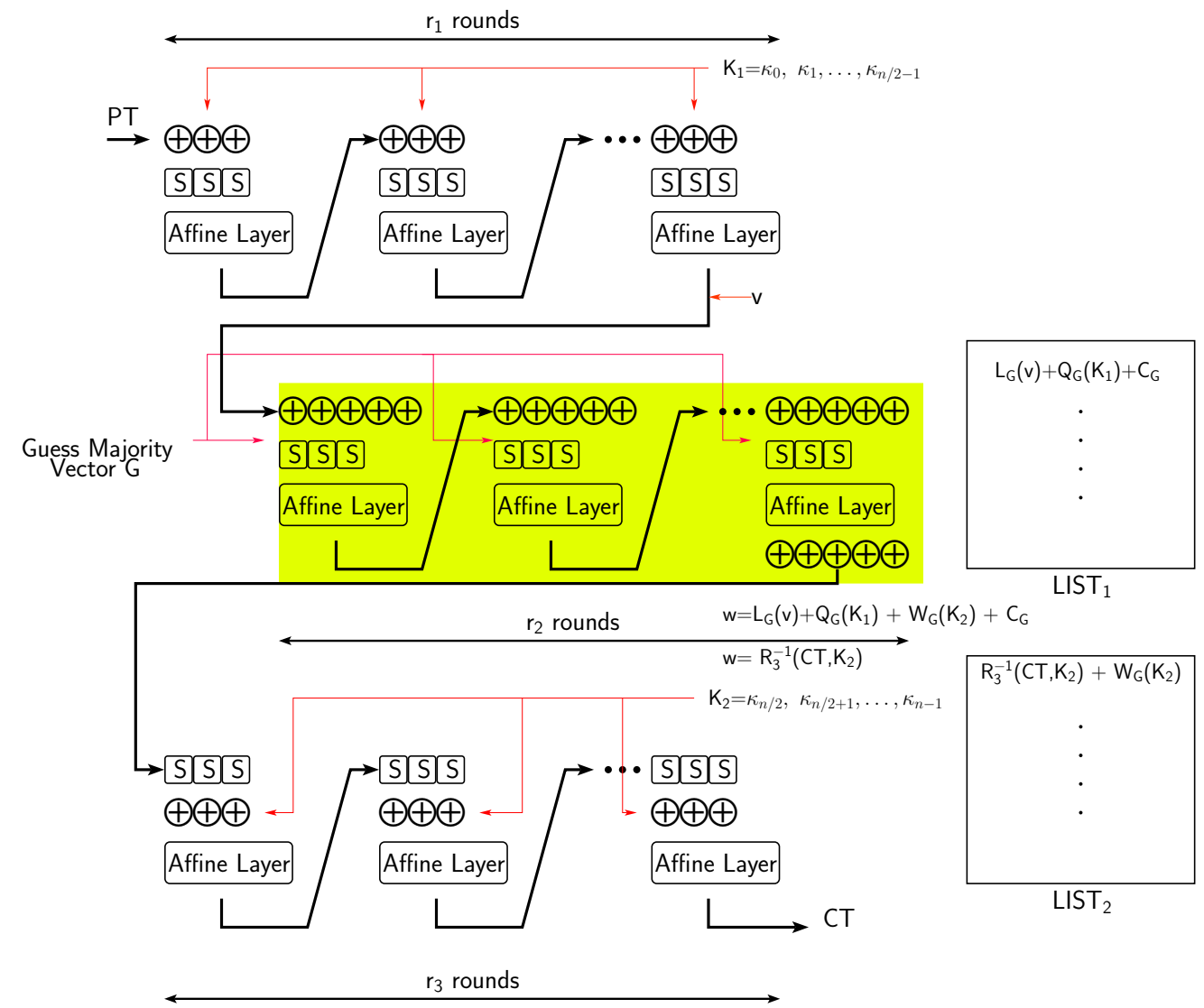

Figure 5: MITM on $r_{1}+r_{2}+r_{3}$ rounds with partial S-box layers. Note that the first $r_{1}$ and last $r_{3}$ rounds have been transformed as per the procedures explained in Figures 3, 4 . We guess the majority bits at the S-box inputs in the middle $r_{2}$ rounds (shown against yellow background) so that they become affine.

see that all the keybits in the middle $r_{2}=0.8\left\lfloor\frac{n}{s}\right\rfloor-\left\lfloor\frac{n}{3 s}\right\rfloor$ rounds can be written as linear functions of $\kappa_{0}, \kappa_{1}, \ldots, \kappa_{n-1}$. Now let us divide the keybits into $K_{1}=\left[\kappa_{0}, \kappa_{1}, \ldots, \kappa_{n / 2-1}\right]$ and $K_{2}=\left[\kappa_{n / 2}, \kappa_{n / 2+1}, \ldots, \kappa_{n-1}\right]$ where $K_{1}$ and $K_{2}$ are the keybits used in the first $r_{1}$ and the final $r_{3}$ rounds respectively.

Now if all the $s \cdot r_{2}$ majority bits of the middle $r_{2}$ rounds are guessed, then the transformation in the middle $r_{2}$ rounds becomes completely affine. If $G$ is the vector of these $s \cdot r_{2}$ majority bits, let us denote this affine transformation in the middle rounds as $L_{G}(x)+Q_{G}\left(K_{1}\right)+W_{G}\left(K_{2}\right)+C_{G}$, where $L_{G}$ is a linear function from $\{0,1\}^{n} \rightarrow\{0,1\}^{n}$ and $Q_{G}, W_{G}$ are linear functions over $\{0,1\}^{n / 2} \rightarrow\{0,1\}^{n}$ and $C_{G}$ is an $n$-bit constant. Let $v$ be the $n$-bit vector obtained by executing the $r_{1}$ forward rounds by guessing some value of $K_{1}$, and let $w$ be the vector obtained after $r_{1}+r_{2}$ rounds. Then after guessing $G$ we have $w=L_{G}(v)+Q_{G}\left(K_{1}\right)+W_{G}\left(K_{2}\right)+C_{G}$. Now $w$ can also be obtained by guessing $K_{2}$ and executing the inverse of the final $r_{3}$ rounds on the ciphertext. If $R_{3}$ denotes the transformation in the last $r_{3}$ rounds, we have $w=R_{3}^{-1}\left(c t, K_{2}\right)$ So we have $R_{3}^{-1}\left(c t, K_{2}\right)=L_{G}(v)+Q_{G}\left(K_{1}\right)+W_{G}\left(K_{2}\right)+C_{G}$. Rearranging terms we have

$$
R_{3}^{-1}\left(c t, K_{2}\right)+W_{G}\left(K_{2}\right)=L_{G}(v)+Q_{G}\left(K_{1}\right)+C_{G}
$$

Then our meet in the middle algorithm will proceed as follows.

1. Guess the vector $G$ of the $s \cdot r_{2}$ majority values in the middle rounds. Find the 
functions $L_{G}, W_{G}, K_{G}$ and $C_{G}$. This step is done $2^{s \cdot r_{2}}$ times in the worst case.

- For all possible values of $K_{2}$, create a hash table LIST $_{2}$ indexed by the $n$-bit vector $R_{3}^{-1}\left(c t, K_{2}\right)+W_{G}\left(K_{2}\right)$. We need $2^{n / 2}$ operations in this step.

- For all possible values of $K_{1}$, create a hash table LIST $_{1}$ indexed by the $n$-bit vector $L_{G}(v)+Q_{G}\left(K_{1}\right)+C_{G}$. We need $2^{n / 2}$ operations in this step.

- Find a collision between $\operatorname{LIST}_{1}$ and $\mathrm{LIST}_{2}$.

- When a collision is found for $K_{1}$ and $K_{2}$ check if the majority bits are consistent with the guess of the key. If yes, this key is in fact the encryption key. Otherwise try another guess of $G$.

The procedure has been explained diagrammatically in Figure 5. Again as explained before, 2 hash tables are not necessary in practice. The attacker can insert each new vector of LIST $_{1}$ and LIST 2 in a single hash table and wait till a collision between elements of LIST and $\mathrm{LIST}_{2}$. The majority of the computational complexity is taken by the guessing of $G$ and computing $R_{3}^{-1}\left(c t, K_{2}\right)+W_{G}\left(K_{2}\right)$ for each guess of $K_{2}$ and $L_{G}(v)+Q_{G}\left(K_{1}\right)+C_{G}$ for each guess of $K_{1}$. This part takes $2^{s \cdot r_{2}} \cdot 2^{1+n / 2} \approx 2^{s \cdot r-n / 3+n / 2}=2^{r s+n / 6}$. For $r=0.8\left\lfloor\frac{n}{s}\right\rfloor$, this complexity is around $2^{29 n / 30}$.

\subsection{When all the key expressions $\kappa_{i}, i \in[0, n-1]$ are not linearly independent}

Note that each $\kappa_{i}$ is a linear expression in the $n$ master key bits, and so it may turn out that the $n$ linear expressions for $\kappa_{i}, i \in[0, n-1]$ are not linearly independent. Assuming each $\kappa_{i}$ is a random linear expression, the probability that they are linearly independent is the same as the probability that a random $n \times n$ matrix over $G F(2)$ is invertible. In fact it is a well known result in discrete mathematics, that this probability is around 0.29 as $n$ becomes large.

When all the $\kappa_{i}$ 's are not linearly independent, then we can not write the round keys in the middle $r_{2}$ rounds as linear expressions of the $\kappa_{i}$ 's. And if this happens, then naturally the attack as outlined in the previous subsection can not be applied. In that case how do you proceed with the attack?

1. Let us assume that for some $r_{1}, r_{3}$, the total rank of the $3 \cdot s \cdot\left(r_{1}+r_{3}\right) \times n$ matrix containing the linear expressions (in terms of the master key) for all the keybits $\kappa_{i}$ used in these rounds be equal to $\lambda$. We have already seen that that when $3 \cdot s \cdot\left(r_{1}+r_{3}\right)=n$, the probability that $\lambda=n$ is 0.29 . The probability that $\lambda=t$ is given by the expression $2^{-n \cdot t} \prod_{i=0}^{t-1}\left(1-\frac{2^{i}}{2^{n}}\right)$. Therefore the probability that $t \geq n-1, n-2, n-3$ is around $0.58,0.77,0.88$ respectively (for large enough $n$ ).

2. In such an event the attacker should choose suitable values of $r_{1}, r_{3}$ such that the value of $\lambda=3 \cdot s \cdot\left(r_{1}+r_{3}\right)$.

3. Let $K=\left[\kappa_{0}, \kappa_{1}, \ldots, \kappa_{\lambda-1}\right]$ be the corresponding keybits whose linear expressions are linearly independent. Let $K_{1}$ be the subset of these keybits used in the first $r_{1}$ rounds, $K_{2}$ be the subset of these keybits used in the last $r_{3}$ rounds. Choose $K_{3}=\left[\kappa_{\lambda}, \kappa_{\lambda+1}, \ldots, \kappa_{n-1}\right]$ as random linear expressions of the master key such that the expressions for $K_{1}, K_{2}, K_{3}$ are linearly independent. After this step all round keybits can be written as linear expressions in $K_{1}, K_{2}, K_{3}$.

4. After guessing $G$, the vector of the middle $s \cdot r_{2}$ majority bits, the middle $r_{2}$ rounds become completely affine. Again if $v$ is the vector that is the output of the first $r_{1}$ rounds, the output vector $w$ of the first $r_{1}+r_{2}$ rounds can be written 
as $w=L_{G}(v)+Q_{G}\left(K_{1}\right)+W_{G}\left(K_{2}\right)+E_{G}\left(K_{3}\right)+C_{G}$, where $L_{G}, Q_{G}, E_{G}$ are linear functions and $C_{G}$ is an $n$-bit constant.

5. Since $w$ can be computed from the ciphertext backwards as $w=R_{3}^{-1}\left(c t, K_{2}\right)$ So we have $R_{3}^{-1}\left(c t, K_{2}\right)=L_{G}(v)+Q_{G}\left(K_{1}\right)+W_{G}\left(K_{2}\right)+E_{G}\left(K_{3}\right)+C_{G}$. Rearranging terms we have $R_{3}^{-1}\left(c t, K_{2}\right)+W_{G}\left(K_{2}\right)=L_{G}(v)+Q_{G}\left(K_{1}\right)+E_{G}\left(K_{3}\right)+C_{G}$. Let us partition $K_{3}$ into two disjoint sets $K_{31}$ and $K_{32}$ so that the number of bits in $K_{1} \cup K_{31}$ and $K_{2} \cup K_{32}$ are almost same. We write $E_{G}\left(K_{3}\right)=E_{G}^{1}\left(K_{31}\right)+E_{G}^{2}\left(K_{32}\right)$. Rearranging terms further we have

$$
R_{3}^{-1}\left(c t, K_{2}\right)+W_{G}\left(K_{2}\right)+E_{G}^{2}\left(K_{32}\right)=L_{G}(v)+Q_{G}\left(K_{1}\right)+E_{G}^{1}\left(K_{31}\right)+C_{G}
$$

After this our meet in the middle algorithm will proceed as follows.

1. Choose suitable values of $r_{1}, r_{3}$ such that the value of $3 \cdot s \cdot\left(r_{1}+r_{3}\right)=\lambda$.

2. Choose $K_{3}=\left[\kappa_{\lambda}, \kappa_{\lambda+1}, \ldots, \kappa_{n-1}\right]$ as random linear expressions of the master key such that the expressions for $K_{1}, K_{2}, K_{3}$ are linearly independent.

3. Partition $K_{3}$ into two disjoint sets $K_{31}$ and $K_{32}$ so that the number of bits in $K_{1} \cup K_{31}$ and $K_{2} \cup K_{32}$ are almost same.

4. Guess the vector $G$ of the $s \cdot r_{2}$ majority values in the middle rounds. Find the functions $L_{G}, W_{G}, K_{G}, E_{G}^{1}, E_{G}^{2}$ and $C_{G}$. This step is done $2^{s \cdot r_{2}}$ times in the worst case.

- For all possible values of $K_{2} \cup K_{32}$, create a hash table $\mathrm{LIST}_{2}$ indexed by the $n$-bit vector $R_{3}^{-1}\left(c t, K_{2}\right)+W_{G}\left(K_{2}\right)+E_{G}^{2}\left(K_{32}\right)$. We need around $2^{n / 2}$ operations in this step.

- For all possible values of $K_{1} \cup K_{31}$, create a hash table LIST $_{1}$ indexed by the $n$-bit vector $L_{G}(v)+Q_{G}\left(K_{1}\right)+E_{G}^{1}\left(K_{31}\right)+C_{G}$. We need around $2^{n / 2}$ operations in this step.

- Find a collision between $\operatorname{LIST}_{1}$ and $\mathrm{LIST}_{2}$.

- When a collision is found check if the majority bits are consistent with the guess of the key. If yes, this key is in fact the encryption key. Otherwise try another guess of $G$.

Again the majority of the computational complexity is taken by the guessing of $G$ and computing $R_{3}^{-1}\left(c t, K_{2}\right)+W_{G}\left(K_{2}\right)+E_{G}^{2}\left(K_{32}\right)$ for each guess of $K_{2} \cup K_{32}$ and $L_{G}(v)+$ $Q_{G}\left(K_{1}+E_{G}^{1}\left(K_{31}\right)\right)+C_{G}$ for each guess of $K_{1} \cup K_{31}$. This part takes $2^{s \cdot r_{2}} \cdot 2^{1+n / 2}$. If $r_{1}+r_{3}=\left\lfloor\frac{n}{3 s}\right\rfloor-\Delta$ then the complexity can be rewritten as $2^{s \cdot r+s \cdot \Delta-n / 3+n / 2}=2^{s r+s \Delta+n / 6}$. For $r=0.8\left\lfloor\frac{n}{s}\right\rfloor$, this complexity is around $2^{29 n / 30+s \Delta}$. Thus the procedure becomes a valid attack if and only if $s \Delta<n / 30$. Thus since $\Delta$ is at least 1 when the first and last keybits are not all linearly independent, the procedure does not work for all challenge instances when $s=10$.

\section{Improving Complexities using the 3-xor problem}

The 3-xor problem in a nutshell is as follows: given 3 lists $L_{1}, L_{2}, L_{3}$ of binary strings over $\{0,1\}^{n}$, the task is to find 3 elements $x_{1} \in L_{1}, x_{2} \in L_{2}, x_{3} \in L_{3}$ such that $x_{1} \oplus x_{2} \oplus x_{3}=0$. This problem has been extensively studied in the literature. Wagner studied in [Wag02], the generalized k-xor problem and showed that for the 4-xor problem if we have lists of size $2^{n / 3}$ then a solution can be found in time $O\left(2^{n / 3}\right)$. However the 3 -xor problem 
still required $O\left(2^{n / 2}\right)$ time using his approach. In [Nan15] a forgery attack was mounted against the COPA mode of operation requiring only $2^{n / 3}$ encryption queries and about $2^{2 n / 3}$ time. This attack was later refined in [NS15], using an improved 3-xor algorithm, to $2^{n / 2-\epsilon}$ queries and $2^{n / 2-\epsilon}$ )operations, for small $\epsilon$. In [LS19], the authors attacked the 2 -round Even Mansour algorithm using this problem with data and time both lower than $2^{n}$. However, the algorithm we use was proposed by Joux [Jou09, Section 8.3.3.1], which is the best algorithm for the 3-xor problem to this day. A generalization for the above algorithm for variable sized lists was proposed in [BDF18], however since we will use lists of fixed size in this section, Joux's algorithm is more relevant here.

Before we discuss the details of the attack it is best to summarize the algorithm in a few words. We begin with the following lemma.

Lemma 2. Given $n / 2$ randomly generated vectors over $\{0,1\}^{n}$, then with high probability they are linearly independent.

Proof. The above probability is given by $p=2^{-n^{2} / 2} \cdot \prod_{i=0}^{n / 2-1}\left(2^{n}-2^{i}\right)$. For large $n$, this equals

$$
p=\prod_{i=0}^{n / 2-1}\left(1-\frac{2^{i}}{2^{n}}\right) \approx 1-\frac{\sum_{i=0}^{n / 2-1} 2^{i}}{2^{n}}=1-\frac{2^{n / 2}-1}{2^{n}} \approx 1-2^{-n / 2}
$$

The algorithm proceeds with 3 lists $L_{1}, L_{2}, L_{3}$ of size $2^{n / 2} / \ell, 2^{n / 2} / \ell, \ell^{2}$ respectively where $\ell=\sqrt{n / 2}$. The list $L_{3}$ has $n / 2$ random vectors which span at most a subspace of rank $n / 2$. It is possible to choose vectors $\mathbb{B}=\left\{b_{1}, b_{2}, \ldots, b_{n / 2}, b_{n / 2+1}, b_{n / 2+2}, \ldots, b_{n}\right\}$ such that all vectors in $L_{3}$ belong to the subspace generated by $b_{n / 2+1}, b_{n / 2+2}, \ldots, b_{n}$. Now designate $M$ to be the $n \times n$ binary matrix that changes the basis of all vectors in $L_{1}, L_{2}, L_{3}$ to $\mathbb{B}$. Note that in the modified basis all elements in $L_{3}$ will begin with $n / 2$ zeros. From the previous lemma, we know that the elements in $L_{3}$ are linearly independent with very high probability. In that case $b_{n / 2+1}, b_{n / 2+2}, \ldots, b_{n}$ can be simply taken as the elements of $L_{3}$ which ensures that in the modified basis the elements of $L_{3}$ have hamming weight exactly equal to 1 , i.e. it has 1 in one of the positions from $n / 2+1$ to $n$. Note that if there exists 3 vectors $x_{1} \in L_{1}, x_{2} \in L_{2}, x_{3} \in L_{3}$ such that $x_{1} \oplus x_{2} \oplus x_{3}=0$, then $M x_{1} \oplus M x_{2} \oplus M x_{3}=0$ for any $n \times n$ binary matrix $M$. Once $M$ is fixed, it can be used to transform $L_{1}$ and $L_{2}$. After this, all we need to do is to search for pairs of elements $\left(x_{1}, x_{2}\right) \in L_{1} \times L_{2}$ such that $M \cdot x_{1} \oplus M \cdot x_{2}$ equals 0 on the first $n / 2$ bits, (and when all the vectors in $L_{3}$ are linearly independent we simply have to check if the sum has hamming weight 1) and this of course can be done efficiently in the following way.

1. After transforming all elements of $L_{3}$ in the new basis $\mathbb{B}$, insert the elements in hash table $J$.

2. After transforming all elements of $L_{1}$ in the new basis $\mathbb{B}$, insert the elements in hash table $H$ indexed by first $n / 2$ bits. All cells of the table should be able to hold multiple elements.

3. After transforming all elements of $L_{2}$ in the new basis $\mathbb{B}$, insert the elements in the same hash table $H$ indexed by first $n / 2$ bits. Note that if any cell of $H$ has more than one elements then their sum in the first $n / 2$ bits must be 0 . By standard randomness assumptions there will be $\frac{2^{n+1} \cdot 2^{-n / 2}}{n}=\frac{2^{n / 2+1}}{n}$ such pairs left whose sum needs to be tested for membership in $L_{3}$.

4. Assuming that testing for membership in $J$ can be done in constant time, we need $\frac{2^{n / 2+1}}{n}$ tests. Note that most of the time $L_{3}$ is linearly independent and so testing for membership in $L_{3}$ can be done by simply checking whether the hamming weight of 
the full vector is 1 , and whether it begins with $n / 2$ zeros. In this case, it is neither necessary to change the basis of vectors in $L_{3}$ nor store them anywhere.

Since the complexity of preparing each list is $O\left(2^{n / 2} / \sqrt{n / 2}\right)$ and around $O\left(2^{n / 2+1} / n\right)$ membership tests are required the complexity of the algorithm is $O\left(2^{n / 2+1} / \sqrt{n / 2}+\right.$ $\left.2^{n / 2+1} / n\right) \approx O\left(2^{n / 2+1} / \sqrt{n / 2}\right)$. This gives a speedup of around $\sqrt{n / 2}$ compared to the basic birthday algorithm of Wagner.

\subsection{MITM on 2-round full S-box layer}

The improved algorithm closely follows the one presented in Sec 5.1 earlier. The basic idea is still the same: this time we partition the key $K$ into 3 sets $K_{1}=\left\{k_{0}, k_{1}, k_{2}, \ldots, k_{m-1}\right\}, K_{2}=$ $\left\{k_{m}, k_{m+1}, k_{m+2}, \ldots, k_{2 m-1}\right\}$ and $K_{3}=\left\{k_{2 m}, k_{2 m+1}, \ldots, k_{n-1}\right\}$, where the value of $m$ is given by $\left\lfloor\log _{2}\left(2^{n / 2} / \sqrt{n / 2}\right)\right\rfloor$, and so the size of $K_{3}$ is considerably smaller and only around $\left\lfloor\log _{2}(n / 2)\right\rfloor$.

Our strategy will be, as before, to guess the $s$ majority bits $\eta_{1}, \ldots, \eta_{s}$ at the input of the second round inverse S-boxes to linearize $R_{2}$ (and of course its inverse). Borrowing the terminology from Sec 5.1, where $x$ denotes the $n$-bit input to the second round and $R K_{1}, R K_{2}$ denote the first, second round keys which are linear functions of the original key $K=R K_{0}$, we have $R_{1}\left(p t+R K_{0}, R K_{1}\right)=x$ and $R_{2}\left(x, R K_{2}\right)=c t$. Since after guessing the majority bits $\eta_{I}$ the inverse of $R_{2}$ becomes linear, we can write each bit $x_{i}$ of $x$ as $x_{i}=A_{i}\left(K_{1}\right)+B_{i}\left(K_{2}\right)+C_{i}\left(K_{3}\right)+d_{i}, \forall i \in[0, n-1]$, where all $A_{i}, B_{i}, C_{i}$ are linear functions and $d_{i}$ is a constant.

Similarly in $R_{1}$, the set of keybits in $K_{1}, K_{2}, K_{3}$ can be partitioned in a manner so that they are not combined multiplicatively in the first round. Hence computing $R_{1}$ in the forward direction from the plaintext input it is possible to write each $x_{i}$ as $f_{i}\left(K_{1}\right)+g_{i}\left(K_{2}\right)+h_{i}\left(K_{3}\right)+e_{i}, \forall i \in[0, n-1]$, where all $f_{i}, g_{i}, h_{i}$ are quadratic functions and $e_{i}$ is a constant. Equating these expressions we have $A_{i}\left(K_{1}\right)+B_{i}\left(K_{2}\right)+C_{i}\left(K_{3}\right)+d_{i}=$ $f_{i}\left(K_{1}\right)+g_{i}\left(K_{2}\right)+h_{i}\left(K_{3}\right)+e_{i}$. Rearranging terms we have:

$$
\underbrace{\left[A_{i}\left(K_{1}\right)+f_{i}\left(K_{1}\right)+d_{i}\right]}_{L_{1}}+\underbrace{\left[B_{i}\left(K_{1}\right)+g_{i}\left(K_{1}\right)+e_{i}\right]}_{L_{2}}+\underbrace{\left[C_{i}\left(K_{3}\right)+h_{i}\left(K_{3}\right)\right]}_{L_{3}}=0
$$

Note that if 3 lists are enumerated for the terms in the square braces, then we arrive exactly at the scenario of the 3 -xor problem. We need to find 3 elements form these lists that sum to 0 . So our modified algorithm will be as follows:

1. Calculate the functional forms of $f_{i}, g_{i}, h_{i}$ and $e_{i}$ for all $i \in[0, n-1]$.

2. Guess the values $\eta_{1}, \ldots, \eta_{s}$. This step is done $2^{s}$ times in the worst case.

- Compute $A_{i}, B_{i}, C_{i}, d_{i}$ for all $i \in[0, n-1]$ using the guessed values.

- For all possible values of $K_{3}$, create a hash table $L_{3}$ indexed by the $n$-bit vector $\left[C_{i}\left(K_{3}\right)+h_{i}\left(K_{3}\right)\right], \forall i \in[0, n-1]$. From here find the matrix $M$ that would transform basis the basis $\mathbb{B}=\left\{b_{1}, b_{2}, \ldots, b_{n / 2}, b_{n / 2+1}, b_{n / 2+2}, \ldots, b_{n}\right\}$ such that $L_{3}$ is spanned by $b_{n / 2+1}, b_{n / 2+2}, \ldots, b_{n}$. With high probability the list $L_{3}$ is linearly independent so that $b_{n / 2+1}, b_{n / 2+2}, \ldots, b_{n}$ can be taken to be the vectors in $L_{3}$. Multiply all vectors in $L_{3}$ by $M$ and store in a hash table $J$. Note there are around $n / 2$ steps here.

- For all possible values of $K_{1}$, create a hash table $L_{1}$ indexed by the $n$-bit vector $M \cdot\left[A_{i}\left(K_{1}\right)+f_{i}\left(K_{1}\right)+d_{i}\right], \forall i \in[0, n-1]$. We need $2^{n / 2} / \sqrt{n / 2}$ operations in this step. 
- For all possible values of $K_{2}$, create a hash table $L_{2}$ indexed by the $n$-bit vector $M \cdot\left[B_{i}\left(K_{1}\right)+g_{i}\left(K_{1}\right)+e_{i}\right], \forall i \in[0, n-1]$. We need $2^{n / 2} / \sqrt{n / 2}$ operations in this step.

- Note that in practice, 2 different hash tables are not necessary. We can instead use one single hash table $H$ in which all elements of $L_{1}, L_{2}$ are inserted indexed by the first $n / 2$ bits as explained in the previous subsection.

- For all pairs in $x_{1}, x_{2} \in H$ which are in the same cell

A: Discard if the sum is not in $L_{3}$. For most cases this can easily be verified by checking if the hamming weight of the sum is 1 , i.e. if $L_{3}$ is linearly independent.

- Once a solution for $K_{1}, K_{2}$ and $K_{3}$ is found, check if the majority bits are consistent with the guess of the key. If yes, this key is in fact the encryption key. Otherwise try another guess of $\eta_{1}, \ldots, \eta_{s}$.

For each majority guess, the complexity of the attack is dominated by finding a collision between two lists of length $O\left(2^{n / 2} / \sqrt{n / 2}\right)$. So the total complexity of the attack is $O\left(2^{s} \times 2 \cdot 2^{n / 2} / \sqrt{n / 2}\right)=O\left(n^{-1 / 2} \cdot 2^{s+n / 2+1}\right)$. This gives a speed up of around $\sqrt{n / 2}$ over the attack in Section 5.1.

There are some further issues to be discussed. We ideally want the lists $L_{1}$ and $L_{2}$ of the same size, but it is often not possible due to the algebraic structure of LowMC. Since we have to partition the keybits such that the cardinality of each set should be a multiple of 3 , it is not always possible to get lists of size $2^{n / 2} / \sqrt{n / 2}, 2^{n / 2} / \sqrt{n / 2}$ and $n / 2$. For $n=129$ we have $n / 2=64.5 \approx 2^{6}$, and so we can take $K_{3}$ to be the last 6 bits of the key, and $K_{1}$ and $K_{2}$ may contain the first 60 and the next 63 bits of the key respectively. In that case, the cost of preparing the lists is around $2^{60}+2^{63} \approx 2^{63}$. The sum of the transformed vectors in $L_{1}$ and $L_{2}$ would need to be zero in the first $129-64=65$ bits and so after filtering $2^{60+63-65} \approx 2^{58}$ vector sums need to be tested for membership in $L_{3}$. So the total cost is around $2^{63}+2^{60}+2^{58} \approx 2^{63}$. Multiplying this by the $2^{43}$ times we need to guess majority bits, this comes to $2^{63+43}=2^{106}$, which results in a speed up of factor 8 compared to the basic MITM in Section 5.1. For $n=192$, we have $n / 2=96 \approx 2^{6.58}$. The only feasible choice of the size of $K_{3}$ is again 6 , which forces $K_{1}$ and $K_{2}$ to be of size 93 each. The cost of preparing lists is around $2^{93}+2^{93}=2^{94}$. However the number of pairs needed to be tested for membership in $L_{3}$ is $2^{93+93-(192-64)}=2^{58}$. So the total complexity for list matching is around $2^{94}+2^{58} \approx 2^{94}$. Multiplying by the number of majority guesses, we get the total complexity as $2^{64+94}=2^{158}$ which also results in a speed up of 8 compared to Section 5.1. Similarly for $n=255$, we have to take $K_{1}, K_{2}, K_{3}$ of sizes $123,126,6$ respectively. A similar calculation yields the total complexity as $2^{85+126}=2^{211}$ which results again in a speedup of 8 compared to the basic MITM.

\section{Conclusion}

In this paper we describe attacks on instances of LowMC where the number of S-boxes is less than the security level, when we use only one plaintext/ciphertext pair. A cryptanalysis of this kind is important as it results in a forgery on the post-quantum signature scheme PICNIC. Since our attacks are in the KPA/KCA scenario and since we use only one plaintext/ciphertext pair, it is not possible to apply traditional symmetric cryptanalytic techniques like differential, linear or any other higher order differential attacks. We begin by showing how to efficiently linearize the LowMC S-box by guessing only one single balanced quadratic expression in its input bits. We leverage this fact to present two types of attacks. First is a simple linearization attack where the attacker obtains a set of linear equations on the key bits relating the plaintext and ciphertext. The second is a meet in 
the middle attack, which takes advantage of the fact that in a single LowMC round, all key bits are not combined multiplicatively. We then show how to improve the attack on the 2-round full S-box layer variant of LowMC with the help of Joux's algorithm to solve the 3 -xor problem.

\section{Acknowledgments}

We wish to thank Hadi Soleimany for helping us improve this draft. Subhadeep Banik was supported by the Swiss National Science Foundation (SNSF) through the Ambizione Grant PZ00P2_179921. Khashayar Barooti was supported by the Swiss National Science Foundation (SNSF) through the project grant 192364 on Post Quantum Cryptography.

\section{References}

[ARS $\left.{ }^{+} 15\right]$ Martin R. Albrecht, Christian Rechberger, Thomas Schneider, Tyge Tiessen, and Michael Zohner. Ciphers for MPC and FHE. In Advances in Cryptology - EUROCRYPT 2015 - 34th Annual International Conference on the Theory and Applications of Cryptographic Techniques, Sofia, Bulgaria, April 26-30, 2015, Proceedings, Part I, pages 430-454, 2015.

[BDF18] Charles Bouillaguet, Claire Delaplace, and Pierre-Alain Fouque. Revisiting and improving algorithms for the 3xor problem. IACR Trans. Symmetric Cryptol., 2018(1):254-276, 2018.

$\left[\mathrm{CDG}^{+} 17\right]$ Melissa Chase, David Derler, Steven Goldfeder, Claudio Orlandi, Sebastian Ramacher, Christian Rechberger, Daniel Slamanig, and Greg Zaverucha. Postquantum zero-knowledge and signatures from symmetric-key primitives. In Proceedings of the 2017 ACM SIGSAC Conference on Computer and Communications Security, CCS 2017, Dallas, TX, USA, October 30 - November 03, 2017, pages 1825-1842, 2017.

[DEM15] Christoph Dobraunig, Maria Eichlseder, and Florian Mendel. Higher-order cryptanalysis of lowmc. In Information Security and Cryptology - ICISC 2015 - 18th International Conference, Seoul, South Korea, November 25-27, 2015, Revised Selected Papers, pages 87-101, 2015.

[DKRS] Christoph Dobraunig, Daniel Kales, Chistian Rechberger, and Markus Schofnegger. Survey of key-recovery attacks onlowmc in a single plaintext/ciphertext scenario. https://raw.githubusercontent.com/ lowmcchallenge/lowmcchallenge-material/master/docs/survey ·pdf.

[IKOS07] Yuval Ishai, Eyal Kushilevitz, Rafail Ostrovsky, and Amit Sahai. Zeroknowledge from secure multiparty computation. In Proceedings of the 39th Annual ACM Symposium on Theory of Computing, San Diego, California, USA, June 11-13, 2007, pages 21-30, 2007.

[Jou09] Antoine Joux. Algorithmic Cryptanalysis. CRC Press, 2009.

[LIM20] Fukang Liu, Takanori Isobe, and Willi Meier. Cryptanalysis of Full LowMC and LowMC-M with Algebraic Techniques. IACR Cryptol. ePrint Arch., 2020:1034, 2020.

[LS19] Gaëtan Leurent and Ferdinand Sibleyras. Low-memory attacks against tworound even-mansour using the 3-xor problem. In Advances in Cryptology - 
CRYPTO 2019 - 39th Annual International Cryptology Conference, Santa Barbara, CA, USA, August 18-22, 2019, Proceedings, Part II, pages 210-235, 2019 .

[Nan15] Mridul Nandi. Revisiting security claims of XLS and COPA. IACR Cryptol. ePrint Arch., 2015:444, 2015.

[nis] Nist post quantum cryptography project. https://csrc.nist.gov/Projects/ post-quantum-cryptography/round-3-submissions.

[NS15] Ivica Nikolic and Yu Sasaki. Refinements of the k-tree algorithm for the generalized birthday problem. In Advances in Cryptology - ASIACRYPT 2015 - 21st International Conference on the Theory and Application of Cryptology and Information Security, Auckland, New Zealand, November 29 - December 3, 2015, Proceedings, Part II, pages 683-703, 2015.

[RST18] Christian Rechberger, Hadi Soleimany, and Tyge Tiessen. Cryptanalysis of low-data instances of full lowmcv2. IACR Trans. Symmetric Cryptol., 2018(3):163-181, 2018.

[Wag02] David A. Wagner. A generalized birthday problem. In Moti Yung, editor, Advances in Cryptology - CRYPTO 2002, 22nd Annual International Cryptology Conference, Santa Barbara, California, USA, August 18-22, 2002, Proceedings, volume 2442 of Lecture Notes in Computer Science, pages 288-303. Springer, 2002. 